\title{
Measurements of heavy flavour forward-backward asymmetries at LEP I
}

\author{
Peter Hansen* \\ Niels Bohr Institute \\ Blegdamsvej 17 \\ DK-2100 Copenhagen $O$ \\ phansen@nbi.dk
}

ABSTRACT: New measurements of $A_{\mathrm{FB}}^{b}$ from the ALEPH and DELPHI experiments are presented at this conference. They significantly reduce the error on the LEP average pole asymmetry, which becomes:

corresponding to

$$
A_{\mathrm{FB}}^{b, 0}=0.0990 \pm 0.0017
$$

$$
\sin ^{2} \theta_{\mathrm{W}}^{\mathrm{eff}}=0.23226 \pm 0.00031
$$

In this presentation, two new measurements using inclusive $b$-hadron final states are discussed.

\section{Methods}

The measurements of the $b$ quark forward-backward asymmetry at the $\mathrm{Z}$ pole provide the most precise determination of $\sin ^{2} \theta_{\mathrm{W}}$ at LEP, hence the interest in improving these measurements. Significant improvements have been achieved by ALEPH and DELPHI with three new results submitted to this conference: A final measurement using inclusive $b$ hadron final states from ALEPH [iin, an updated preliminary measurement also using $b$ hadron final states from DELPHI [i2] 2$]$, and a new measurement from DELPHI using $K^{ \pm}$ tagging [i] $\left[\begin{array}{l}3 \\ ]\end{array}\right.$. The two inclusive measurements are discussed here, since they carry the largest statistical weight in the average.

The ALEPH measurement uses the jet charge method in a sample of mostly $Z \rightarrow b \bar{b}$. The difference between the jet charges in the forward and backward hemispheres is:

$$
\left\langle Q_{\mathrm{FB}}\right\rangle=\left\langle Q_{\mathrm{F}}-Q_{\mathrm{B}}\right\rangle=\sum_{f=u, d, s, c, b} P_{f} \delta_{f} A_{\mathrm{FB}}^{f} \frac{8}{3} \frac{\cos \theta}{1+\cos \theta^{2}}
$$

${ }^{*}$ Speaker. 
where $\theta$ is the polar angle of the event axis. The flavour purities, $P_{f}$, are measured in data using double tag fits to the conditional probability density of hemisphere $b$-tags. The charge separations, $\delta_{f}$, are calibrated using fits to measurements of

$$
\bar{\delta}^{2}=\sigma^{2}\left(Q_{\mathrm{FB}}\right)-\sigma^{2}\left(Q_{\mathrm{tot}}\right)=\sum_{f} P_{f} \bar{\delta}_{f}^{2}
$$

where the fitted $\bar{\delta}_{f}$ 's are equal to the $\delta_{f}$ 's within a MC correction from hemisphere charge correlations of about $9 \%$.

The DELPHI measurement uses the counting method for hemispheres with a good secondary vertex:

$$
\frac{N_{\mathrm{F}}^{+}+N_{\mathrm{B}}^{-}-N_{\mathrm{F}}^{-}-N_{\mathrm{B}}^{+}}{N_{\text {tot }}}=\sum_{f=u, d, s, c, b} P_{f}\left(2 * w_{f}-1\right) A_{\mathrm{FB}}^{f} \frac{8}{3} \frac{\cos \theta}{1+\cos \theta^{2}}
$$

where $w_{b}$ is the fraction of correctly signed hemispheres, which is calibrated in the selected data using the ratio between opposite-sign and same-sign events:

$$
\frac{N_{\text {opp }}}{N_{\text {same }}} \approx \frac{w_{b}^{2}+\left(1-w_{b}\right)^{2}}{2 w_{b}\left(1-w_{b}\right)}
$$

where, again, the equality holds up to a small MC correction from charge correlations and background.

\section{Flavour and charge tags}

ALEPH uses a neural net hemisphere $b$-tag composed of lifetime information, event shapes and lepton $p_{T}$, extending the analysis to $\cos \theta_{\text {thrust }}<0.95$. A large sample of $670 \mathrm{~K}$ events, of which $88 \%$ are $b \bar{b}$, are chosen on the basis of the $b$-tag. This is a $30 \%$ increase in statistics compared with previous results [i⿱亠幺厶]

DELPHI Uses a $b$-tag based on reconstructed secondary vertices and selects a $96 \%$ pure $b \bar{b}$ sample of about $200 \mathrm{~K}$ events. Here, the small $c$ and light flavor efficiencies are taken from MC. The differences in event selection between ALEPH and DELPHI causes a high degree of independence in the systematic errors.

Both experiments use neural nets to estimate the quark charge in each hemisphere. Typical inputs to the nets are:

- Jet charges, secondary and primary vertex charges (for various weighting powers $\kappa$ ).

- Information from particle ID (note that lepton-ID is not used in ALEPH, and only indirectly in DELPHI. This is in order to minimize correlations with the measurements based on leptonic final states.) 
- Secondary vertex quality, estimated $b$-hadron momentum and lifetime and other items intended to optimize the weight of each charge estimator according to the event topology.

Different strategies are used to combine the information in the two experiments, but in each case they enhance the correct sign probability by $\approx 10 \%$ over the simple jet-charge estimate.

\section{Systematic errors}

As mentioned, the differences in event selection give rise to rather different systematic errors in the two experiments as illustrated by the Table below. In ALEPH the purities and charge resolutions, also for non- $b$ flavours, are found from fits to data. Thus, the uncertainties due to flavour composition are of statistical nature. The uncertainty due to charge correlations is unusually small in ALEPH. It comes from varying the assumed heavy flavour parameters in the MC. Assumptions regarding light quark and gluon hadronization have not been varied, however. Instead a series of comparisons between data and MC final states are used to estimate the uncertainty due to fragmentation effects. A check is provided by the charge separation in events with a high $p_{T}$ lepton, since the lepton-ID information is not used in building the hemisphere charges. The sign of the lepton charge then provides independent information on the forward-backward orientation of the event, and by comparing the signed charge separation in data and $\mathrm{MC}$, the predicted charge correlation is confirmed within a $0.7 \%$ statistical precision.

DELPHI uses a very pure sample of $b \bar{b}$ and therefore the contribution to the error from $A_{\mathrm{FB}}^{c}$ is relatively small, whereas other contributions are relatively large. A check is in the DELPHI case provided by the correct sign probability in events with an identified $D^{*}$. This confirms the assumed value within a statistical error of $3.6 \%$.

\begin{tabular}{|l|c|c|}
\hline \multicolumn{1}{|c|}{ Source of systematic uncertainty $\Delta A_{\mathrm{FB}}^{b}$} & ALEPH & DELPHI prel. \\
\hline \hline flavor purities and quark charges & 0.00083 & 0.00155 \\
\hline$b$-hadron production and decay parameters & 0.00014 & 0.00021 \\
\hline$c$-hadron production and decay parameters & 0.00019 & 0.00017 \\
\hline charge correlations & 0.00028 & 0.00120 \\
\hline $\mathrm{QCD}$ effects & 0.00019 & 0.00042 \\
\hline$A_{\mathrm{FB}}^{c}, R_{b}$ and $R_{c}$ & 0.00047 & 0.00014 \\
\hline Detector resolution & 0.00023 & 0.00056 \\
\hline \hline Total systematic error on $A_{\mathrm{FB}}^{b}$ & \pm 0.0012 & \pm 0.0021 \\
\hline
\end{tabular}

\section{Results}

Summarizing the measurements of the forward-backward b-asymmetry at LEP, we have:

New final inclusive result $(\mathrm{ALEPH})$ 檕:

$$
A_{\mathrm{FB}}^{b, 0}=0.1011 \pm 0.0027 \text { (stat) } \pm 0.0012 \text { (syst) }
$$


New preliminary inclusive result (DELPHI) [2]2] :

$$
A_{\mathrm{FB}}^{b, 0}=0.0997 \pm 0.0036(\text { stat }) \pm 0.0021 \text { (syst) }
$$

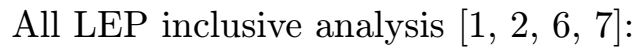

$$
A_{\mathrm{FB}}^{b, 0}=0.1009 \pm 0.0020
$$

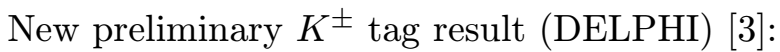

$$
A_{\mathrm{FB}}^{b, 0}=0.1069 \pm 0.0089(\text { stat }) \pm 0.0068 \text { (syst) }
$$

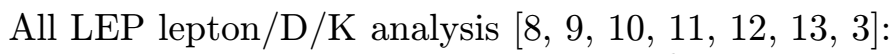

$$
A_{\mathrm{FB}}=0.0968 \pm 0.0026
$$

All LEP and SLD measurements:

$$
\underline{A_{\mathrm{FB}}^{b, 0}=0.0990 \pm 0.0017}
$$

It is interesting to note that:

- The inclusive measurements have been significantly improved over the earlier mea-

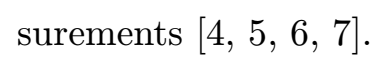

- However, the errors are still statistics dominated.

- There are correlations of $25-30 \%$ between the inclusive and leptonic measurements

- The different LEP measurements are internally consistent within $1.4 \sigma$.

- A $3.2 \sigma$ difference still remains between the $\sin ^{2} \theta_{\mathrm{W}}^{\text {eff }}$ derived from $A_{\mathrm{FB}}^{b}$ and the one derived from the SLD measurement of $A_{\mathrm{LR}}\left[{ }_{1}^{1} \overline{1} \overline{4}\right]$.

Further progress in these measurements would be very wellcome. However, all ongoing analysis are approaching final numbers.

\section{References}

[1] ALEPH Collaboration, "Measurement of $A_{\mathrm{FB}}^{b}$ using Inclusive b-hadron Decays", CERN EP/2001-057, http://alephwww.cern.ch/ALPUB/paper/paper01/6/preprint.ps

[2] DELPHI Collaboration, "Determination of $A_{\mathrm{FB}}^{b}$ using inclusive charge reconstruction and lifetime tagging at LEP1",

http://delphiwww.cern.ch/ ${ }^{\sim}$ pubxx/delsec/conferences/summer01/paper_eps297_lp198.ps.gz

[3] DELPHI Collaboration, "Measurement of the forward-

backward asymmetry of bottom quarks at the Z0 peak using charged kaons for quark-charge tag", http://delphiwww.cern.ch/ pubxx/delsec/conferences/summer01/paper_eps298_lp199.ps.gz

[4] ALEPH Collaboration, "Determination of $A_{\mathrm{FB}}^{b}$ using jet charge measurements in $Z$ decays", Phys. Lett. B426 (1998) 217.

[5] DELPHI Collaboration "Measurement of $A_{F B}^{b \bar{b}}$ in hadronic $Z$ decays using a jet charge technique" Eur. Phys. J. C9 (1999) 367. 
[6] OPAL Collaboration "Measurement of b quark forward backward asymmetry around the $Z^{0}$ peak using jet charge and vertex charge", Z. Phys. C75 (1997) 385.

[7] L3 Collaboration, "Measurement of the $e^{+} e^{-} \rightarrow b \bar{b}$ and $e^{+} e^{-} \rightarrow c \bar{c}$ forward-backward asymmetries at the $Z^{0}$ resonance", Phys. Lett. B439 (1998) 225.

[8] ALEPH Collaboration "Measurement of the $b$ forward backward asymmetry and mixing using high-p $p_{T}$ leptons", Phys. Lett. B384 (1996) 414.

[9] DELPHI Collaboration "Measurement of the forward-backward asymmetry of $e^{+} e^{-} \rightarrow Z \rightarrow b \bar{b}$ using prompt leptons", Z. Phys. C65 (1995) 569.

[10] L3 Collaboration "Measurement of the $e^{+} e^{-} \rightarrow Z \rightarrow b \bar{b}$ forward backward asymmetry and the $B^{0}-\bar{B}^{0}$ mixing parameter using prompt leptons", Phys. Lett. B448 (1999) 152.

[11] OPAL Collaboration "Measurement of heavy quark forward backward asymmetries and average B mixing using leptons in multihadronic events", Z. Phys. C70 (1996) 357.

[12] DELPHI Collaboration "Measurement of the forward backward asymmetries of $c$ and $b$ quarks at the $Z$ pole using reconstructed D mesons", Eur. Phys. J. C10 (1999) 219.

[13] OPAL Collaboration "A measurement of charm and bottom forward-backward asymmetries using D mesons at LEP", Z. Phys. C73 (1996) 379.

[14] SLD Collaboration, "A High Precision Measurement of the Left-Right Z Boson Cross-Section Asymmetry." , Phys. Rev. Lett. 84 (2000) 5945. 